\title{
Kappa opioid receptor agonists improve postoperative cognitive dysfunction in rats via the JAK2/STAT3 signaling pathway
}

\author{
$\mathrm{XI} \mathrm{LI}^{*}$, YINGJIE SUN ${ }^{2 *}$, QIANG JIN ${ }^{2}$, DANDAN SONG ${ }^{2}$ and YUGANG DIAO ${ }^{2}$ \\ ${ }^{1}$ Postgraduate Training Base of Jinzhou Medical University in The General Hospital of Northern Theater Command, \\ Jinzhou, Liaoning 121013; ${ }^{2}$ Department of Anesthesia, The General Hospital of Northern Theater Command, \\ Shenyang, Liaoning 110016, P.R. China
}

Received January 15, 2019; Accepted August 5, 2019

DOI: $10.3892 /$ ijmm.2019.4339

\begin{abstract}
Postoperative cognitive dysfunction (POCD) is a common and well-known complication following surgery, particularly cardiopulmonary bypass (CPB) surgery. There are currently no suitable treatments for POCD, which is associated with increased illness and mortality rates. The present study aimed to identify a novel treatment for POCD. The protective effect of kappa opioid receptor (KOR) agonists on POCD in rats following $\mathrm{CPB}$ was determined and the regulatory mechanism of the Janus kinase 2/signal transducer and activator of transcription 3 (JAK2/STAT3) signaling pathway was examined. The rats were randomly divided into five groups: Sham operation (Sham group), CPB operation (CPB group), KOR agonist + CPB (K group), KOR agonist + norbinaltorphimine (nor-BNI) + CPB (NK group), and KOR agonist + JAK2-STAT3 specific pathway inhibitor + CPB (AG group). A water maze test and neurological function scores were used to evaluate POCD. Hematoxylin and eosin staining was used to observe hippocampal neurons. ELISA was used to detect the levels of inflammatory factors, oxidative stress factors and brain injury markers. Immunofluorescence was used to visualize the neurons. TUNEL staining and western blotting were used to detect neuronal apoptosis, and western blotting was also used to detect JAK2/STAT3 pathway-related proteins. The KOR agonists significantly improved POCD. S-100 $\beta$ and NSE detection revealed that KOR agonists alleviated brain damage in CPB rats, and this result was reversed by KOR
\end{abstract}

Correspondence to: Dr Yingjie Sun or Dr Yugang Diao, Department of Anesthesia, The General Hospital of Northern Theater Command, 83 Wenhua Road, Shenyang, Liaoning 110016, P.R. China

E-mail: sunyingjie9@hotmail.com

E-mail:diao72@163.com

*Contributed equally

Key words: kappa opioid receptor agonist, cognitive dysfunction, Janus kinase 2/signal transducer and activator of transcription 3 signaling pathway antagonists. The KOR agonists led to a significantly reduced inflammatory response and oxidative stress, as determined by ELISA detection, and attenuated hippocampal neuronal apoptosis, as revealed by TUNEL staining and western blotting, compared with the results in the CPB group. Finally, the KOR agonists inhibited the expression levels of phosphorylated (p-)JAK2 and p-STAT3, rather than total JAK2 and STAT3, compared with levels in the CPB group. Taken together, KOR agonists improved POCD in rats with CPB by inhibiting the JAK2/STAT3 signaling pathway.

\section{Introduction}

With the rapid development of technology for cardiac surgery, cardiopulmonary bypass (CPB) and anesthesia, the number of cardiac surgical procedures, particularly CPB, in China has significantly increased. Many patients suffer postoperative central nervous system complications (1). $\mathrm{CPB}$ open-heart surgery is complicated by postoperative cognitive dysfunction (POCD) manifesting in neurological and mental disorders, including dysmnesia, disorientation and visual-spatial ability $(2,3)$. POCD has become a leading cause of mortality and disability in patients following CPB open-heart surgery. The need to prevent and treat POCD initiated by $\mathrm{CPB}$ cardiac surgery has hindered the development of cardiac surgery.

Reactive oxygen species (ROS) are chemically reactive chemical species containing oxygen. ROS are implicated in mediating apoptosis, or programmed cell death, and ischemic injury. During CPB, ischemia and perfusion injury can activate neutrophils and lead to excessive ROS. ROS levels can increase markedly, causing damage in numerous cellular molecules, including lipids, proteins and DNA (4). Drugs with antioxidant properties may reduce ROS bursts and oxidative stress, reducing POCD during and following CPB (5).

Kappa opioid receptors (KORs) are important in regulating ischemic brain damage. Studies have confirmed that KORs alleviate brain damage and improve functional recovery in animal models with general and regional cerebral ischemia $(6,7)$. KOR agonists can significantly improve the hippocampal nerve damage caused by ischemia, thereby alleviating cognitive dysfunction (6). Another report shows that KORs are beneficial for the activation of hippocampal 
cholinergic neurons (8). KOR agonists can block the transport of acetylcholine through the KOR-mediated opioid nervous system to improve learning and memory dysfunction (9). However, the specific regulatory mechanisms by which KOR agonists improve cognitive dysfunction remain unclear.

The Janus kinase 2/signal transducer and activator of transcription 3 (JAK2/STAT3) signaling pathway is involved in the anti-inflammatory response following activation of the acetylcholine receptor $(10,11)$. Numerous studies have confirmed that JAK2/STAT3 activation is involved in the anti-apoptotic process following transient focal cerebral ischemia (12). However, the potential effects of KOR agonists on POCD in CPB rats through the JAK2/STAT3 signaling pathway remain to be fully established.

In the present study, a model of POCD was established in CPB rats. Using this model, the effects of KOR agonists on neurological damage, brain damage, inflammation, oxidative stress and neuronal apoptosis were investigated, and the effect on JAK2/STAT3 signaling pathway-related proteins was further analyzed. The present study aimed to identify a novel therapy for POCD in CPB rats, which may provide theoretical and experimental evidence for the treatment of patients with POCD.

\section{Materials and methods}

Experimental animals and groupings. A total of 50 male Sprague-Dawley rats (SPF grade), 6 months old, weighing 350-450 g, were provided by the Animal Experimental Department of the General Hospital of Northern Theater Command [Shenyang, China; license for rodent use: SYXK (Military) 20120007; production license for rodents: SCXK (Military) 20120006] in accordance with The Guide to the Care and Use of Laboratory Animals published by the Canadian Council on Animal Care. All rats were cultivated in individual ventilated cages at $24 \pm 2^{\circ} \mathrm{C}$ ) and $40-70 \%$ humidity in a 12-h light/dark cycle. Standard pelleted chow and drinking water were available ad libitum. All animal protocols were approved by the Experimental Animal Ethics Committee of the General Hospital of Northern Theater Command (no. GHNTC2018018).

The rats were randomly divided into five groups $(n=10)$ : Sham operation (Sham group), CPB surgery (CPB group), KOR agonist (U50488H) + CPB (K group), KOR agonist $(\mathrm{U} 50488 \mathrm{H})+$ norbinaltorphimine (nor-BNI ) + CPB (NK group), KOR agonist (U50488H) + JAK2-STAT3 pathway inhibitor (AG490) + CPB (AG group). In the K group, the rats were administered with an intravenous injection of U50488H $(1.5 \mathrm{mg} / \mathrm{kg}$, cat. no. 0495/25, Tocris Bioscience, Bristol, UK) 30 min before the CPB assay; in the NK group, an intravenous injection of U50488H $(1.5 \mathrm{mg} / \mathrm{kg})$ was administered when rats were catheterized, and nor-BNI ( $2 \mathrm{mg} / \mathrm{kg}$, Sigma-Aldrich; Merck KGaA) was administered intravenously 30 min later; in the AG Group, U50488H $(1.5 \mathrm{mg} / \mathrm{kg})$ was administered intravenously when the rats were anesthetized and catheterized, and AG490 $(5 \mathrm{mg} / \mathrm{kg})$ was injected intravenously $30 \mathrm{~min}$ later. When the water maze test was completed, 7 days after CPB bypass, the rats were anesthetized and catheterized, $5 \mathrm{ml}$ of blood was drawn through the right internal vein, and serum was separated by centrifugation at $500 \mathrm{x} \mathrm{g}$ for $5 \mathrm{~min}$. The samples were stored at $-80^{\circ} \mathrm{C}$ until examination. The bilateral hippocampus was immediately removed, with one side stored at $-80^{\circ} \mathrm{C}$ and the other side fixed in $10 \%$ formalin at room temperature for $48 \mathrm{~h}$.

Preparation of the CPB model. CPB surgery was performed as previously reported (7), with minor modifications. Briefly, the rats were injected intraperitoneally (i.p.) with $4 \%$ chloral hydrate $(300 \mathrm{mg} / \mathrm{kg}$; Shanghai Ziyuan Pharmaceutical Co., Ltd., Shanghai, China) to induce anesthesia (13). During surgery, anesthesia was maintained with isoflurane (MAC $=1.5 \%$, Hangzhou Minsheng Pharmaceutical Co., Ltd., Hangzhou, China). Photopic oral intubation was performed using a $16-G$ intravenous catheter, and animals were mechanically ventilated with a small animal ventilator (frequency, 60 beats $/ \mathrm{min}$; tidal volume, $3 \mathrm{ml} / \mathrm{kg}$; inspiratory to expiratory ratio, 1:1.5) connected to a monitor to observe the heart rate, oxygen saturation and rectal temperature.

The puncture site was sterilized with iodophor (Shandong Lierkang Disinfection Technology Co., Ltd., Dezhou, China), followed by exposure and puncture of the vein. Right femoral vein catheterization (24-G) was performed to open the fluid path, which was transfused with 6\% hydroxyethyl starch (Guangdong Jiabao Pharmaceutical Co., Ltd., Qingyuan, China) and connected to a microinfusion pump. The left femoral artery was catheterized (22-G) and used to monitor blood pressure. Coccygeal artery catheterization $(22-\mathrm{G})$ and right internal jugular vein catheterization $(18-G)$ were performed to drain blood for CPB. The drainage tube, a homemade blood storage device, a constant peristaltic pump (Baoding Longer Precision Pump Co., Ltd., Baoding, China), silicone tubing (internal diameter, $4 \mathrm{~mm}$ ) and a rat membrane oxygenator (Guangdong Kewei Medical Instrument Co. Ltd., Dongguan, China) were installed between the two puncture sites to establish the CPB circuit. Heparin sodium (300 IU/kg; Shenyang Haitong Pharmaceutical Co., Ltd., Shenyang, China) was injected into the left femoral vein once the activated clotting time reached $480 \mathrm{sec}$.

$\mathrm{CPB}$ was performed with the membrane oxygenator to supply oxygen. The low-flow CPB velocity was $35 \mathrm{ml} / \mathrm{kg} / \mathrm{min}$, which was later increased to $100-120 \mathrm{ml} / \mathrm{kg} / \mathrm{min}$ at full-flow bypass. To prevent air embolism, 1-2 $\mathrm{ml}$ of blood was retained in the blood storage device. The mean arterial pressure was maintained at $>60 \mathrm{mmHg}$, partial $\mathrm{CO}_{2}$ pressure at $35-45 \mathrm{mmHg}$, base excess at $-3-3 \mathrm{mmol} / 1 \mathrm{mmHg}$, $\mathrm{pH}$ at $7.35-7.45$ and hematocrit at $>0.25$. The rats were treated with 2-20 $\mu \mathrm{g} / 100 \mathrm{~g}$ epinephrine hydrochloride (Wuhan Grand Pharmaceutical Group Co., Ltd., Wuhan, China) and fluids during surgery to maintain a stable circulation.

Water maze assessment. After $24 \mathrm{~h}$ of $\mathrm{CPB}$, the water maze test was performed for 7 days consecutively, which included hidden platform tests and space exploration tests. For the hidden platform test, the rats were placed into water from any quadrant facing the pool wall and made to swim for $90 \mathrm{sec}$ to locate the hidden platform. The incubation period of escape was recorded as the time required to locate the hidden platform in the pool. If the platform was not found after $90 \mathrm{sec}$, the rat was directed to the platform and the score was counted as $90 \mathrm{sec}$. The rats were assessed for 5 days, with the first 4 days 
used for training. Any rats with a score of $90 \mathrm{sec}$ were eliminated, and the test scores on day 5 were recorded as the spatial learning and memory scores of the animals.

For the space exploration test, the platform was removed $24 \mathrm{~h}$ after the hidden platform test had ended. The rats were placed in the water at the same place as previously, and their swimming paths were recorded for $60 \mathrm{sec}$. The duration of rats in the original station quadrant and the number of times the original station location was crossed were recorded. The trajectories of the rats were recorded, and information processing was performed using the Morris water maze video analysis system (WMT-100S, Taimeng).

Neurological function scores. After 1, 3, and 7 days of CPB model preparation, the Garcia score scale was used for detecting the neurological functions of the experimental animals (Table I) $(14,15)$.

Hematoxylin and eosin $(H \& E)$ staining. The formalin-fixed tissue samples were placed in 70, 80, 90, 95 and $100 \%$ alcohol. Xylene was used to clear the sample. The samples were embedded into paraffin blocks, cut into $4-\mu \mathrm{m}$ sections and then dewaxed. Hematoxylin staining was performed for $5 \mathrm{~min}$ at room temperature, following which the slides were washed in PBS, immersed in $1 \%$ hydrochloric acid, stained with eosin for $30 \mathrm{sec}$ and then dehydrated in gradient alcohol. Neutral gum was used for sealing. Pathological changes in each group of tissues were observed under a light microscope.

TUNEL assay. The in situ cell death detection kit (Roche Diagnostics GmbH, Mannheim, Germany) was used according to the manufacturer's instructions: The 5- $\mu \mathrm{m}$ sections of paraffin-embedded hippocampal tissue were de-paraffinized by dimethylbenzene for twice, $10 \mathrm{~min}$ per time, permeabilized by gradient elution of alcohol $(100,95,90,80$ and $70 \%)$. The sections were treated with $50 \mu 1$ TUNEL reaction solution for $60 \mathrm{~min}$ in a humid dark box at $37^{\circ} \mathrm{C}$. Subsequently, $50 \mu \mathrm{l}$ of streptavidin-HRP working solution was added to the sections in the dark box for $30 \mathrm{~min}$. The nuclei were fluorescently stained with DAPI, followed by conventional dehydration, decolorization and fixation. The apoptotic rates were examined and images were captured using a light microscope (Olympus Corporation, Tokyo, Japan) at a magnification of $\mathrm{x} 400$, and densitometric scanning was analyzed using the MetaMorph BX41 image analysis system (Olympus Corporation). A total of five images were captured randomly for each section at x400 magnification and integral optical density was calculated using the microscopic image analyzer (MetaMorph BX41 image analysis system). The total nuclei and TUNEL-positive nuclei were counted, and the proportions of TUNEL-positive cells above the number in the untreated controls were calculated as follows: $\%$ apoptosis $=($ number of TUNEL-positive cells/total cells) x100.

ELISA assessment. ELISA kits were used to detect inflammatory factors IL-1 $\beta$ (cat. no. CSB-E08055r, CUSABIO, Wuhan, China), IL-6 (cat. no. SEA079Ra, USCN, Wuhan, China), TNF- $\alpha$ (cat. no. SEA133Si, USCN) and IL-10 (cat. no. SEA056Ra, USCN) in rat serum, stress indicators superoxide dismutase (SOD; cat. no. SES134Hu, USCN),

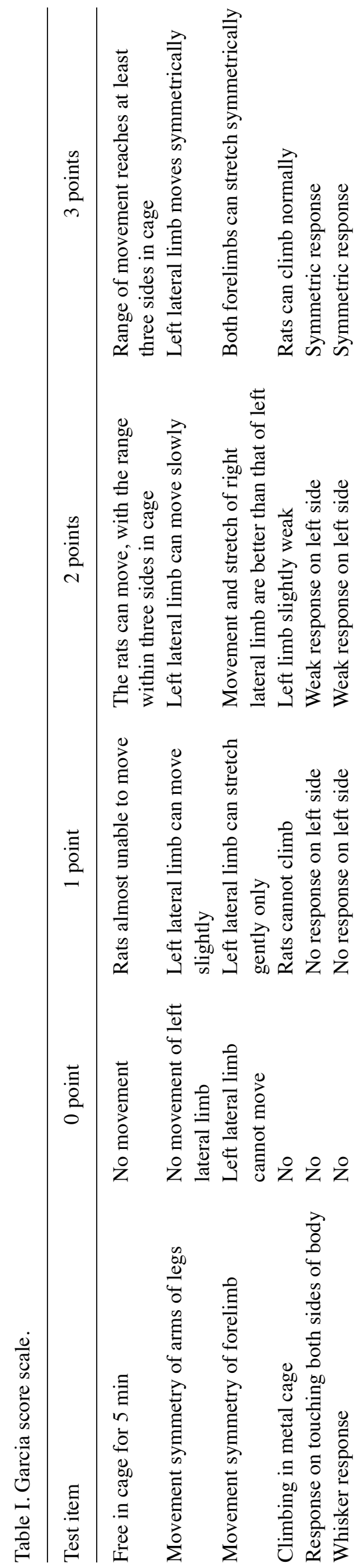


malondialdehyde (MDA; cat. no. CEA597Ge, USCN) and nitric oxide (NO; cat. no. IS100, USCN), and brain damage markers S-100 $\beta$ (cat. no. SEA567Ra, USCN) and neuron-specific enolase (NSE; cat. no. SEA537Ra, USCN). The kit was equilibrated to room temperature and the required reaction plate was removed. Subsequently, $100 \mu 1$ of the standard product and $100 \mu \mathrm{l}$ of the diluted sample were successively added to the well of the corresponding reaction plate, the plate was mixed by gently shaking for $30 \mathrm{sec}$ and then incubated for $20 \mathrm{~min}$ at room temperature. The reaction plate was washed with a washing machine, following which $100 \mu \mathrm{l}$ serum was added to each well and incubated at $37^{\circ} \mathrm{C}$ for $2 \mathrm{~h}$. The plate was washed, and $100 \mu \mathrm{l} \mathrm{HRP-labeled} \mathrm{secondary}$ antibody provided in the kit was added per well and incubated at $37^{\circ} \mathrm{C}$ for $30 \mathrm{~min}$. The plate was washed and $50 \mu \mathrm{l}$ each of color developing solutions A and B were added for $15 \mathrm{~min}$ in the dark, following which $50 \mu \mathrm{l}$ of stop solution was added. The optical density (OD) value at $450 \mathrm{~nm}$ was read on a microplate reader (EXL808 BioTek Instruments, Inc., Winooski, VT, USA).

Using the OD value as the vertical coordinate and the standard concentration as the horizontal coordinate allowed for a standard curve to be drawn and the curve equation and $r$ value to be calculated to determine the corresponding concentration values of each sample.

Immunofluorescence. The paraffin-embedded hippocampal tissues were dewaxed and placed into water and then immersed in 3\% hydrogen peroxide solution for $15 \mathrm{~min}$ and washed with PBS. Subsequent antigen recovery was performed with $0.1 \mathrm{M}$ sodium citrate solution. The tissues were blocked with goat serum (cat. no. SL038, Beijing Solarbio Science \& Technology) at $37^{\circ} \mathrm{C}$ for $30 \mathrm{~min}$ and the serum was then decanted without washing. phosphorylated (p-)JAK2 (1:100, cat. no. ab32101, Abcam) and p-STAT3 (1:100, cat. no. ab76315, Abcam) antibodies were added for incubation overnight at $4^{\circ} \mathrm{C}$. The following day, the sections were washed in PBS and then incubated with Goat Anti-Rabbit IgG H\&L (Cy3 ${ }^{\circledR}, 1: 500$, cat. no. ab6939) antibody at $37^{\circ} \mathrm{C}$ for $30 \mathrm{~min}$ and then washed again with PBS. DAPI dye at $300 \mu \mathrm{M}$ was added for $10 \mathrm{~min}$ at room temperature, following which the tissues were washed with PBS, sealed with neutral gum, and observed under a fluorescence microscope.

Western blotting. Following homogenization of the hippocampus, pre-cooled RIPA (Thermo Fisher Scientific, Inc., Waltham, MA, USA, cat. no. 89900) lysate was added and was lysed on ice for $30 \mathrm{~min}$. Following collection of the supernatant, the concentration of the collected protein solution was determined using a BCA (Thermo Fisher Scientific, Inc., cat. no. 23225) protein quantification kit. The proteins (30 $\mu \mathrm{g} /$ well) were then separated by $12 \%$ SDS-PAGE electrophoresis and transferred onto a PVDF membrane. The membranes were blocked with 5\% skim milk at room temperature for $1.5 \mathrm{~h}$. JAK2 (1:2,000, cat. no. ab108596, Abcam), p-JAK2 (1:2,000, cat. no. ab32101, Abcam), STAT3 (1:2,000, cat. no. ab119352, Abcam), p-STAT3 (1:2,000, cat. no. ab76315, Abcam), Bc1-2 (1:1,000, cat. no. ab59348, Abcam), Bax (1:2,000, cat. no. ab32503, Abcam), pro-caspase-3 (1:1,000, cat. no. ab32150, Abcam) and cleaved caspase 3 (1:500, cat. no. ab49822, Abcam) primary antibodies were added for incubation overnight at $4^{\circ} \mathrm{C}$. Following washing with PBS, Goat Anti-Rabbit IgG H\&L (HRP, 1:10,000, cat. no. ab6721) antibody was added and incubated for $2 \mathrm{~h}$ at room temperature before developing ECL luminescence. A gel imaging system (Gel Doc ${ }^{\mathrm{TM}}$ XR; Bio-Rad Laboratories, Inc) was used for capturing images. Absorbance values were analyzed using ImageJ (v1.8.0; National Institutes of Health).

Statistical analysis. Statistical analyses were performed using SPSS 19.0 (IBM Corp., Armonk, NY, USA) software. Multiple comparisons were analyzed with one-way analysis of variance, followed by an appropriate multiple comparison test (Tukey's procedure). $\mathrm{P}<0.05$ was considered to indicate a statistically significant difference.

\section{Results}

Successful preparation of the CPB rat model. Compared with rats in the Sham group, there were no significant changes in rectal temperature, $\mathrm{pH}$, partial pressure of carbon dioxide $\left(\mathrm{PaCO}_{2}\right)$ or partial pressure of oxygen $\left(\mathrm{PaO}_{2}\right)$ in rats in the CPB group, $\mathrm{K}$ group, NK group and $\mathrm{AG}$ group $(\mathrm{P}<0.05)$, as shown in Fig. 1. Compared with rats in the Sham group, the mean arterial pressure (MAP), heart rate (HR), left ventricular diastolic pressure (LVDP), highest rate of change in pressure development $(+\mathrm{dP} / \mathrm{dtmin})$ and hemoglobin $(\mathrm{Hb})$ were decreased in the CPB group, and this effect was significantly reversed under KOR agonist treatment in the $\mathrm{K}$ group $(\mathrm{P}<0.05)$ (Fig. 1).

KOR agonists alleviate neurological dysfunction in CPB rats. The Garcia neurological function score of the CPB group was $3.2 \pm 1.1$, which was significantly lower than that of the Sham group $(\mathrm{P}<0.05$; Fig. $2 \mathrm{~A})$. When the KOR agonist was administered, the neurological score of rats in the $\mathrm{K}$ group $(8.9 \pm 1.4 ; \mathrm{P}<0.05)$ was significantly higher than that of rats in the CPB group. The neurological function score of rats in the NK group was significantly lower than that of rats in the $\mathrm{K}$ group $(2.9 \pm 0.9 ; \mathrm{P}<0.05)$. The water maze test was used to judge the cognitive function of the rats (Fig. 2B and C). In the hidden platform training test, the latency in finding the platform in the $\mathrm{CPB}, \mathrm{K}$ and $\mathrm{NK}$ groups were all prolonged compared with that in the Sham group $(\mathrm{P}<0.05)$. The latency of finding the platform in the $\mathrm{K}$ group was significantly shorter compared with that in the CPB group $(\mathrm{P}<0.05)$, whereas the latency of finding the platform in the NK group was significantly prolonged compared with that in the $\mathrm{K}$ group $(\mathrm{P}<0.05)$. In the space exploration experiment, the duration the animal stayed in the original station quadrant and the number of times the original station in the target quadrant was crossed were significantly reduced in the $\mathrm{CPB}, \mathrm{K}$ and $\mathrm{NK}$ groups compared with those in the Sham group $(\mathrm{P}<0.05)$. The duration the rat remained in the original station quadrant and the number of times the original station in the target quadrant was crossed were significantly increased in the $\mathrm{K}$ group compared with those in the CPB group $(\mathrm{P}<0.05)$. In the NK group, there were no significant changes in swimming distance or the duration the rat remained in the target quadrant compared with the $\mathrm{CPB}$ group $(\mathrm{P}>0.05)$, although the time spent in the original 

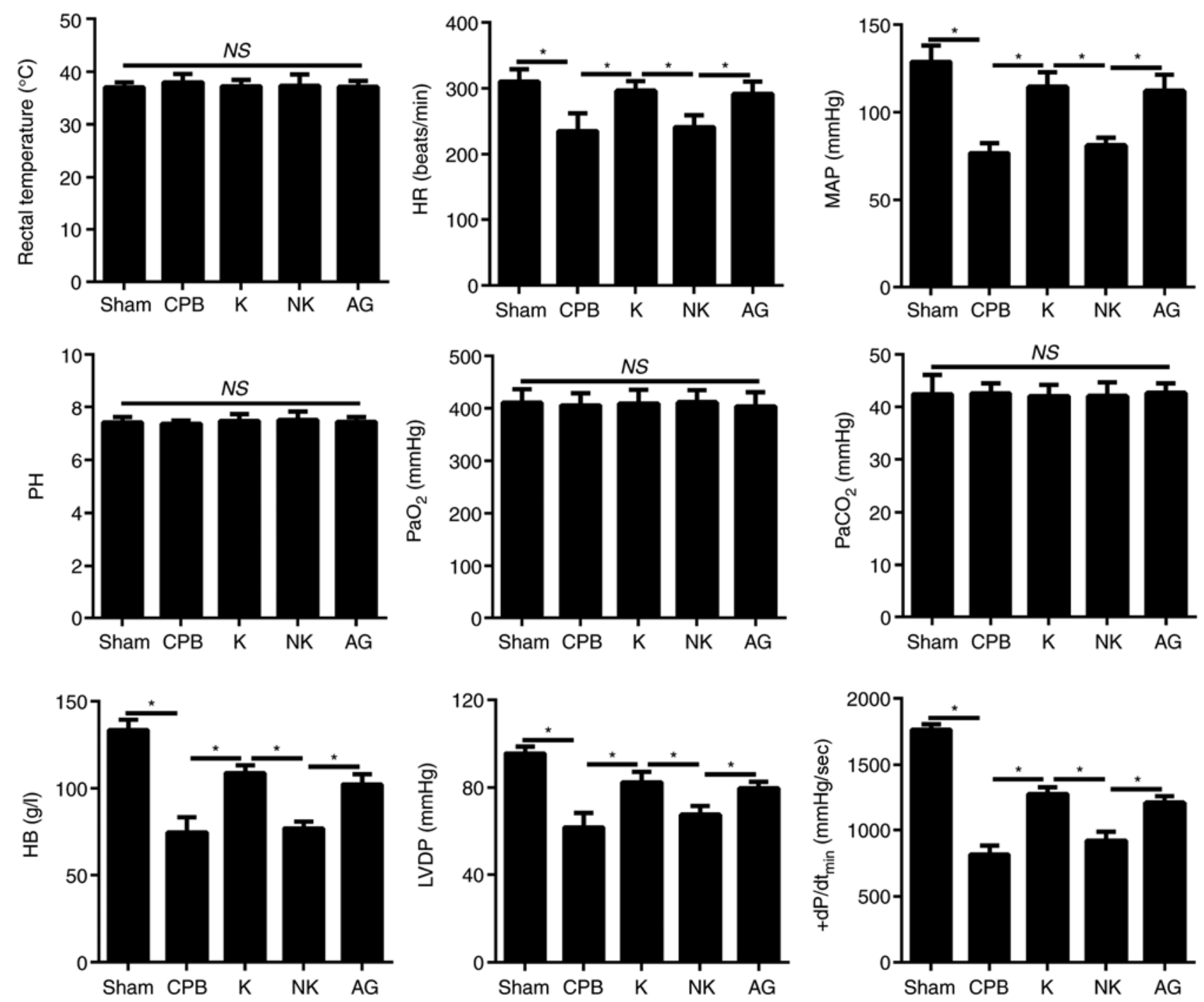

Figure 1. Changes in the hemodynamics of rats. Changes in the rectal temperature, $\mathrm{HR}, \mathrm{MAP}, \mathrm{pH}, \mathrm{PaO}_{2}, \mathrm{PaCO}_{2}, \mathrm{HB}, \mathrm{LVDP}$ and $+\mathrm{dP} / \mathrm{dtmax}$ of rats in each group. ${ }^{*} \mathrm{P}<0.05$. $\mathrm{CPB}$, cardiopulmonary bypass; KOR, kappa opioid receptor; $\mathrm{K}, \mathrm{KOR}$ agonist $+\mathrm{CPB}$; NK, KOR agonist + norbinaltorphimine $+\mathrm{CPB}$; AG, KOR agonist + JAK2-STAT3 specific pathway inhibitor + CPB; KOR, HR, heart rate; MAP, mean arterial pressure; PaO 2 , partial pressure of oxygen; partial pressure of $\mathrm{CO}_{2}$; $\mathrm{HB}$, hemoglobin; LVDP, left ventricular diastolic pressure; ns, not significant.

station quadrant and the number of times the original station was crossed were lower in the NK group compared with those in the $\mathrm{K}$ group $(\mathrm{P}<0.05)$. This suggested that $\mathrm{KOR}$ agonists can alleviate neurological dysfunction in CPB rats.

KOR agonists improve brain dysfunction in $C P B$ rats. As shown in Fig. 3A, H\&E staining revealed that hippocampal neurons in the Sham group were arranged in a regular and tight manner with clear cell boundaries and intact cell bands. The cells were arranged neatly with a normal cell structure. Only a small number of inflammatory cells were present. The hippocampus in the CPB group exhibited severe damage, with disordered cells, widened intercellular spaces and increased astrocyte and vascular proliferation. In the Sham group, the nerve cells in the hippocampal region were normal, arranged regularly, exhibited staining of the cytoplasm, nuclei were round or oval and there were no obvious lesions. In the CPB group, the nerve cells were disordered with nuclei dissolution. Neuronal cell and cone cell death were observed and cell numbers were significantly decreased in the hippocampus. In the $\mathrm{K}$ group, the arrangement of cells in the hippocampus was more regular than that in the CPB group, and the number of degenerative/necrotic nerve cells was significantly lower.

Staining in the $\mathrm{K}$ group revealed less damage than that in the CPB group. The cells were arranged neatly and the cell band was incomplete. The NK group exhibited the most damage. The cells were sparsely and unevenly distributed, and the number of uneven cytoplasmic vacuoles was increased. This suggests that CPB can severely damage the hippocampus of rats, whereas KOR agonists can improve this damage. Compared with the $\mathrm{K}$ group, rats in AG group exhibited notable damage as that in NK group. The cells were scarcely and irregularly distributed and the number of uneven cytoplasmic vacuoles was increased (Fig. 3A). To further examine the brain damage, changes in the expression of brain damage markers were detected by ELISA (Fig. 3B). Compared with those in the Sham group, serum concentrations of NSE and S-100 $\beta$ were increased in the CPB, $\mathrm{K}$ and NK groups $(\mathrm{P}<0.05)$. The serum concentrations of NSE and S-100 $\beta$ were significantly lower in the $\mathrm{K}$ group than in the $\mathrm{CPB}$ group $(\mathrm{P}<0.05)$, whereas 
A

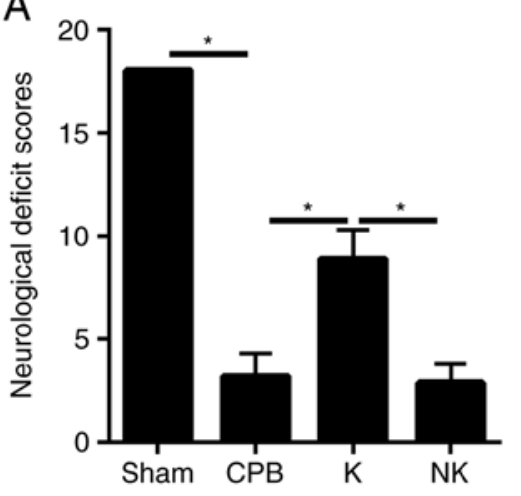

C

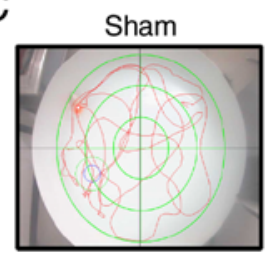

$\mathrm{K}$
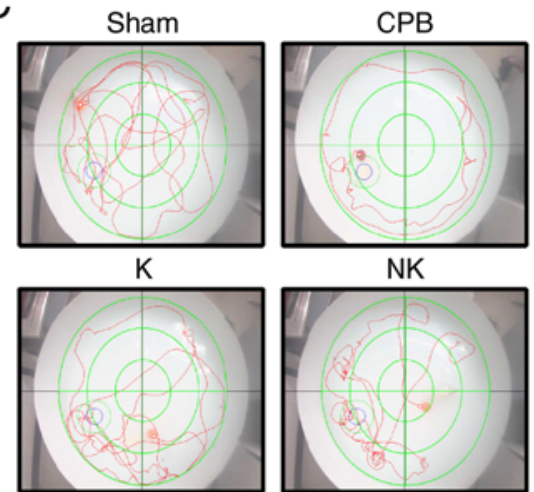

NK

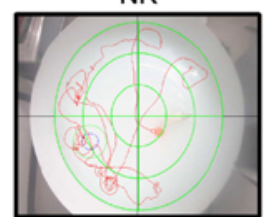

B

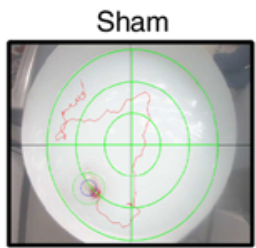

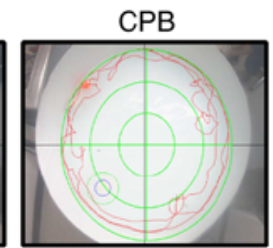
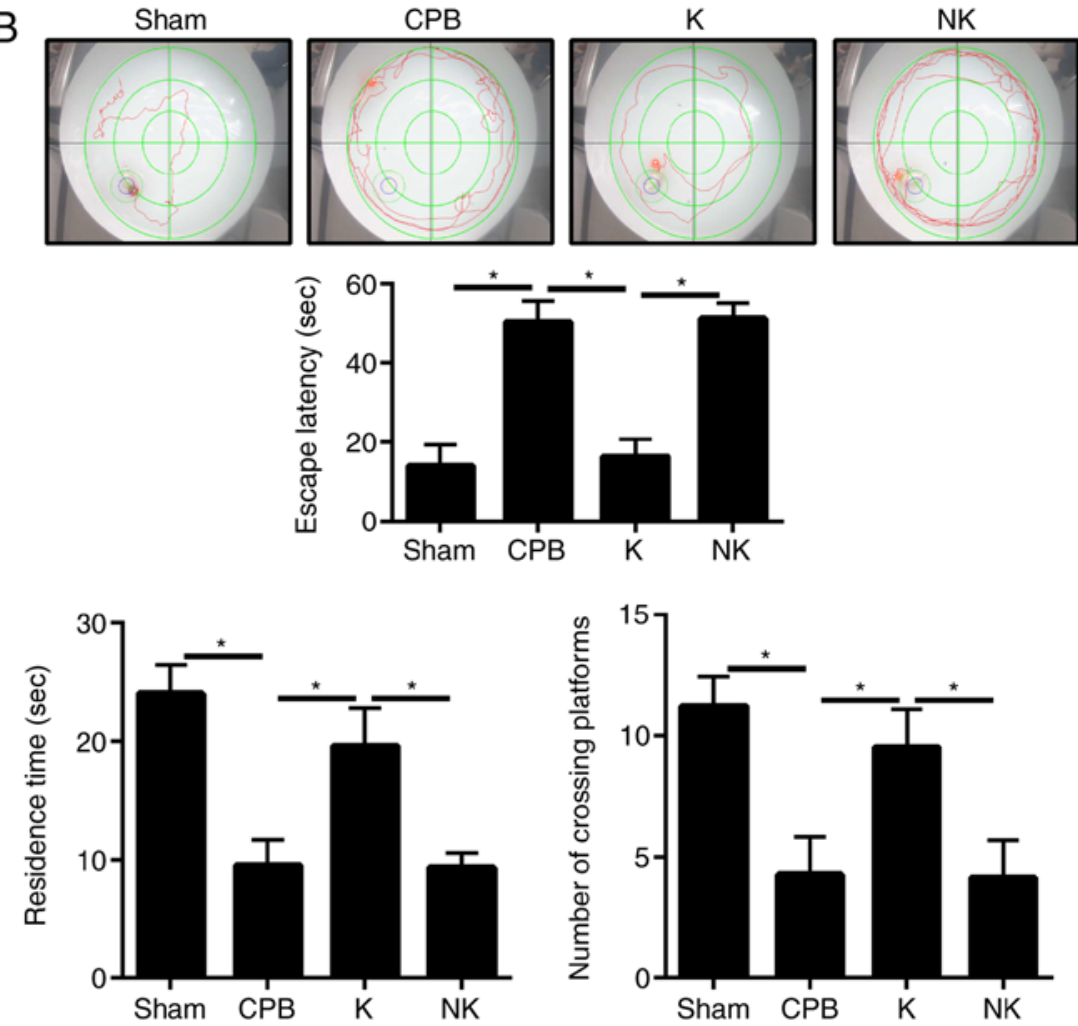

Figure 2. KOR agonists can alleviate neurological dysfunctions in CPB rats. Water maze tests and neurological function scores were used to evaluate postoperative cognitive dysfunction. (A) Neurological function score. (B) Hidden platform training test. (C) Space exploration experiment. The red lines indicated the motion tracking of rats in the water maze test. ${ }^{*} \mathrm{P}<0.05$. CPB, cardiopulmonary bypass; KOR, kappa-opioid receptor; $\mathrm{K}$, KOR agonist $+\mathrm{CPB}$; NK, KOR agonist + norbinaltorphimine $+\mathrm{CPB}$.
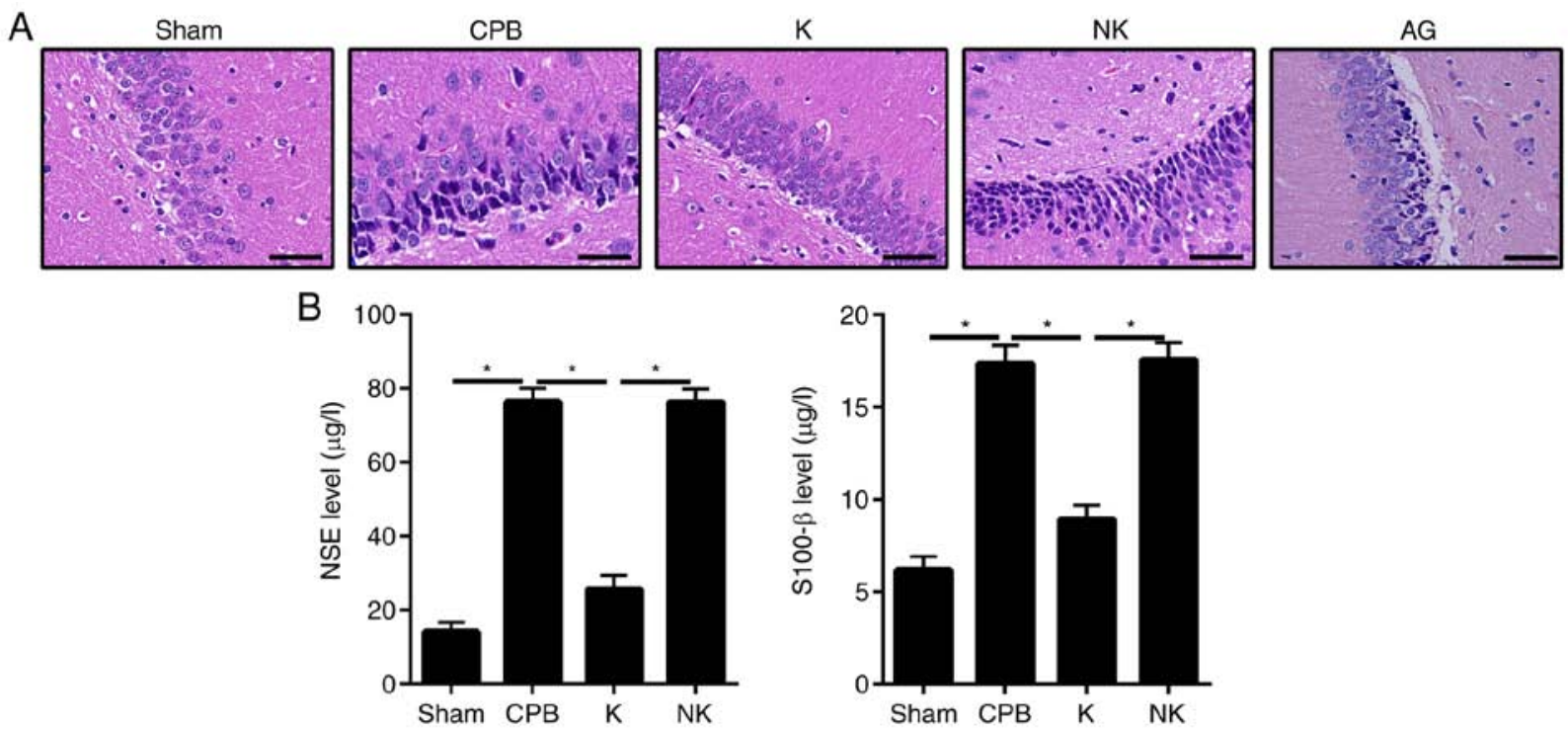

Figure 3. KOR agonists can improve brain dysfunction in CPB rats. H\&E staining was used to observe hippocampal neurons. ELISA was used to detect the levels of brain injury markers. (A) H\&E staining (scale bar=50 $\mu \mathrm{m}$ ). (B) Brain damage markers detected by ELISA; "P $<0.05$. CPB, cardiopulmonary bypass; KOR, kappa opioid receptor; NSE, neuron-specific enolase; K, KOR agonist + CPB; NK, KOR agonist + norbinaltorphimine + CPB; AG, KOR agonist + JAK2-STAT3 specific pathway inhibitor + CPB; AG, KOR agonist + JAK2-STAT3 specific pathway inhibitor + CPB; H\&E, hematoxylin and eosin; JAK2, Janus kinase 2; STAT3, signal transducer and activator of transcription 3.

serum concentrations of NSE and S-100 $\beta$ were significantly higher in the NK and AG group than in the K group $(\mathrm{P}<0.05)$, suggesting that KOR agonists can alleviate brain damage in CPB rats.
KOR agonists inhibit inflammation and oxidative stress in $C P B$ rats. Inflammatory factors (Fig. 4A) and oxidative stress factors (Fig. 4B) in rat serum were tracked by ELISA. The concentrations of IL-1 $\beta$, IL- 6 and TNF- $\alpha$ were increased and 

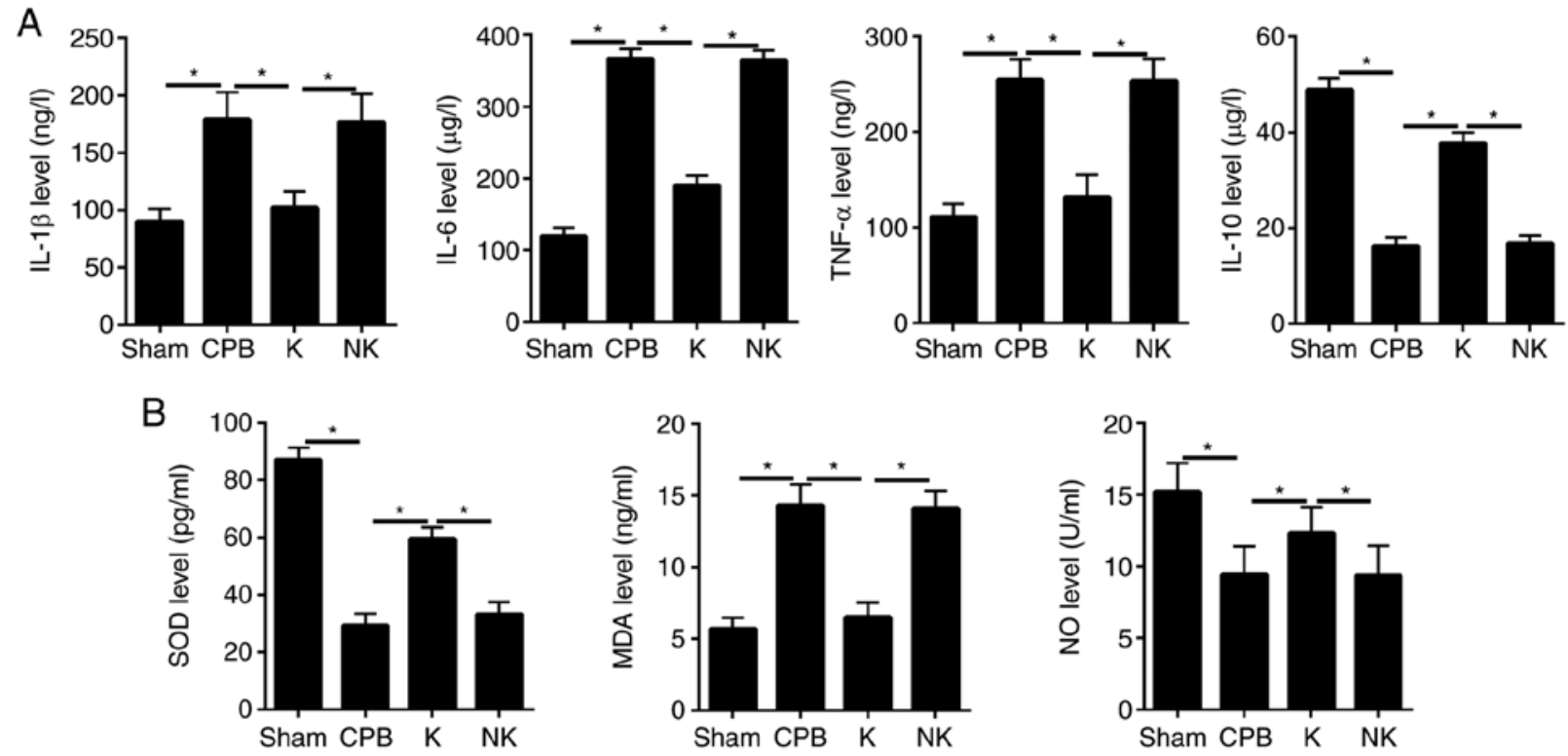

Figure 4. KOR agonists can inhibit inflammation and oxidative stress in CPB rats. ELISA was used to detect the level of inflammatory factors and oxidative stress factors. (A) Inflammatory factors detected by ELISA. (B) Oxidative stress factors detected by ELISA; ${ }^{\text {P }<0.05}$. CPB, cardiopulmonary bypass; KOR, kappa opioid receptor; K, KOR agonist + CPB; NK, KOR agonist + norbinaltorphimine + CPB; SOD, superoxide dismutase; MDA, malondialdehyde; NO, nitric oxide.

that of IL-10 was significantly decreased in the CPB group compared with that in the Sham group. $(\mathrm{P}<0.05)$. Serum concentrations of IL-1 $\beta$, IL- 6 and TNF- $\alpha$ were significantly decreased and that of IL-10 was significantly increased in the $\mathrm{K}$ compared with the that in the $\mathrm{CPB}$ group $(\mathrm{P}<0.05)$. The analysis of oxidative stress factors showed that serum concentrations of SOD and NO were decreased and that of MDA was significantly increased in the CPB group compared with that in the Sham group $(\mathrm{P}<0.05)$. The serum concentrations of SOD and NO were significantly increased, and the concentration of MDA was significantly reduced in the $\mathrm{K}$ group compared with that in the CPB group $(\mathrm{P}<0.05)$. This suggests that $\mathrm{CPB}$ triggered severe inflammation and oxidative stress, both of which were reversed by KOR agonists.

KOR agonists improve neuronal apoptosis in CPB rats. Neuronal apoptosis in the brain tissue was observed by TUNEL staining (Fig. 5A). The number of positive cells in the CPB group was significantly increased compared with that in the Sham group $(\mathrm{P}<0.05)$. The number of positive cells in the hippocampal brain tissue was significantly lower in the $\mathrm{K}$ group than in the CPB group $(\mathrm{P}<0.05)$, whereas the number of positive cells in the NK group was significantly higher than in the $\mathrm{K}$ group $(\mathrm{P}<0.05)$. To detect neuronal apoptosis, the expression of apoptosis-related factors Bcl-2, Bax, pro-caspase-3 and cleaved caspase 3 were detected by western blotting (Fig. 5B). Bcl-2 was significantly decreased and Bax was significantly increased in the CPB group compared with the Sham group $(\mathrm{P}<0.05)$. The expression of $\mathrm{Bcl}-2$ was significantly increased in the $\mathrm{K}$ group compared with that in the $\mathrm{CPB}$ group, whereas the expression of Bax was significantly decreased $(\mathrm{P}<0.05)$. The expression of Bcl-2 was significantly decreased in the NK group compared with that in the $\mathrm{K}$ group, whereas the expression of Bax was significantly increased $(\mathrm{P}<0.05)$. In addition, the expression of pro-caspase-3 was significantly decreased and that of caspase 3 was significantly increased in the CPB group compared with that in the Sham group $(\mathrm{P}<0.05)$. The expression of pro-caspase-3 was significantly increased and that of cleaved caspase 3 was significantly decreased in the $\mathrm{K}$ group compared with that in the $\mathrm{CPB}$ group $(\mathrm{P}<0.05)$. The expression of pro-caspase-3 was significantly decreased and that of cleaved caspase 3 was significantly increased in the NK group compared with that in the $\mathrm{K}$ group $(\mathrm{P}<0.05)$. These results suggest that KOR agonists can inhibit neuronal apoptosis and prevent neuronal degeneration in CPB rats.

Effect of KOR agonist on the expression of JAK2/STAT3 signaling pathway-related proteins in $C P B$ rats. Numerous studies have reported that the JAK2/STAT3 signaling pathway serves an anti-inflammatory role (16) and has anti-apoptotic effects in cerebral ischemic neurons $(17,18)$. Upon treatment with KOR agonists for POCD in CPB rats, western blotting (Fig. 5B) revealed significantly increased levels of JAK2, p-JAK2, STAT3 and p-STAT3 in the hippocampus of the CPB group compared with levels in the Sham group $(\mathrm{P}<0.05)$. The levels of p-JAK2, and p-STAT3 in the hippocampus of the $\mathrm{K}$ group were significantly decreased compared with those in the CPB group, and the levels were significantly higher in the NK group compared with those in the K group $(\mathrm{P}<0.05)$. Immunofluorescence further validated this result (Fig. 5C). The levels of JAK2 and STAT2 were not significantly altered among that in different groups. Therefore, KOR agonists may attenuate POCD in CPB rats through the JAK2/STAT3 signaling pathway.

KOR agonists improve $P O C D$ in $C P B$ rats through the p-JAK2/p-STAT3 rather than JAK2/STAT3. JAK2/STAT3 signaling pathway inhibitors were administered as the AG group, then we identified that and it was found that the levels of hippocampal p-JAK2, p-STAT3, but not the levels of JAK2 and STAT3, were significantly decreased in the $\mathrm{K}$ group compared with those in the CPB group $(\mathrm{P}<0.05)$. Compared with that 
A

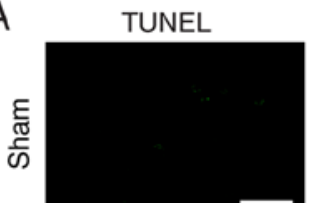

DAPI

Merge
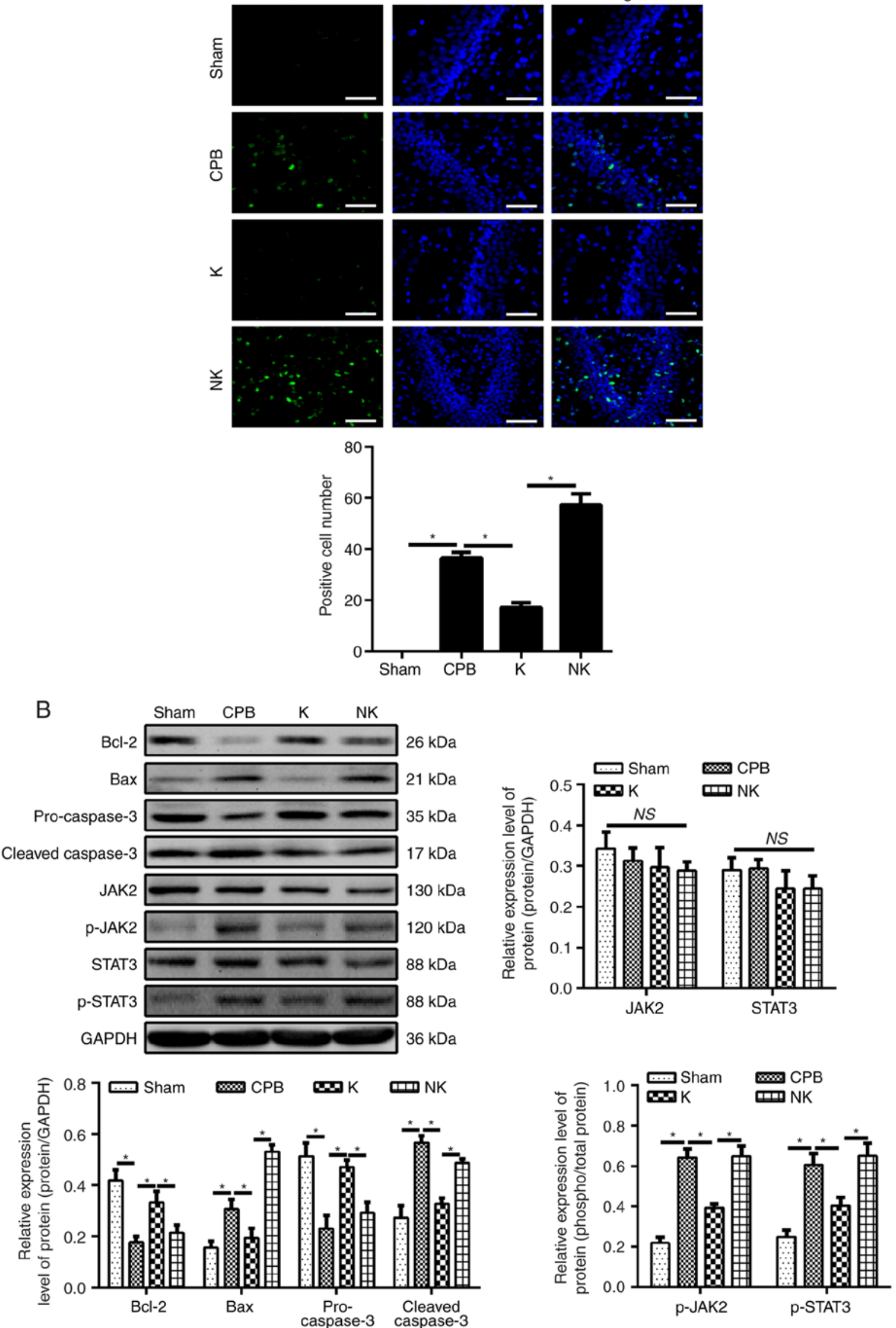

Figure 5. KOR agonists can improve neuronal apoptosis and the effect of KOR agonists via the JAK2/STAT3 signaling pathway in CPB rats. TUNEL staining and western blotting were used to detect apoptotic factors and neuronal apoptosis. Western blotting was used to detect JAK2/STAT3 pathway-related proteins. Immunofluorescence was used to detect p-JAK2 and p-STAT3. (A) TUNEL staining (scale bar=50 $\mu \mathrm{m}$ ). (B) Expression levels of apoptosis-related proteins and JAK2/STAT3 signaling pathway-related proteins were detected by western blotting. 
C
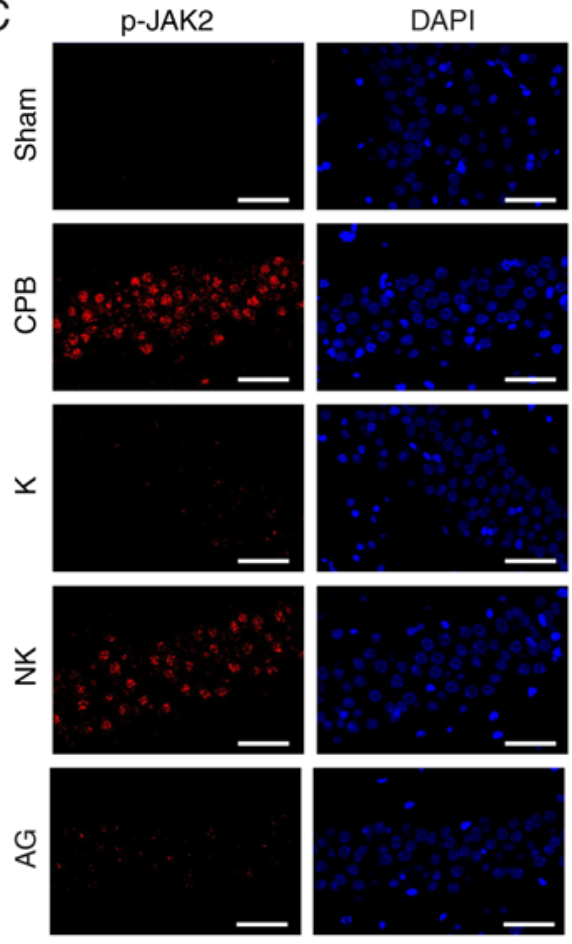

Merge
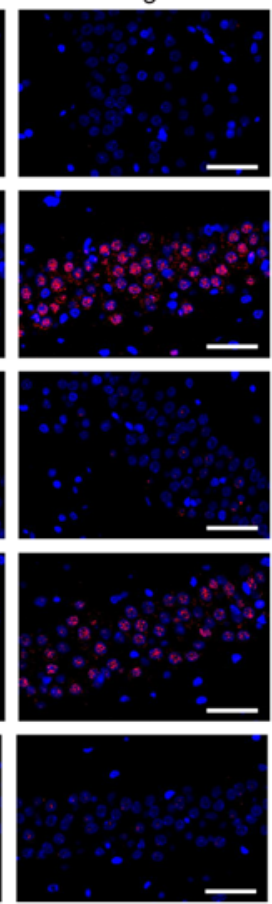

p-STAT3
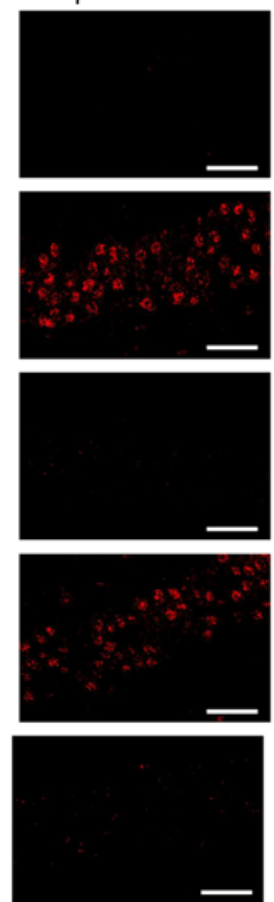

DAPI
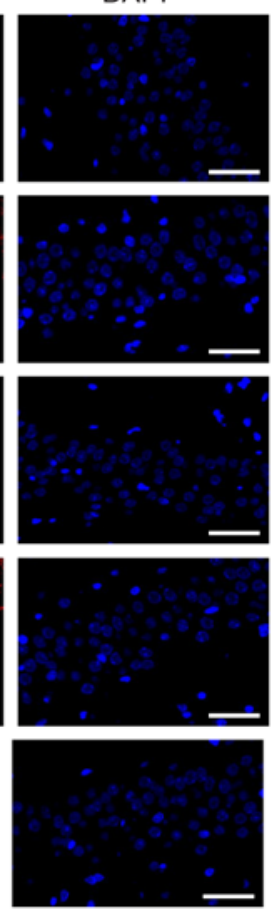

Merge
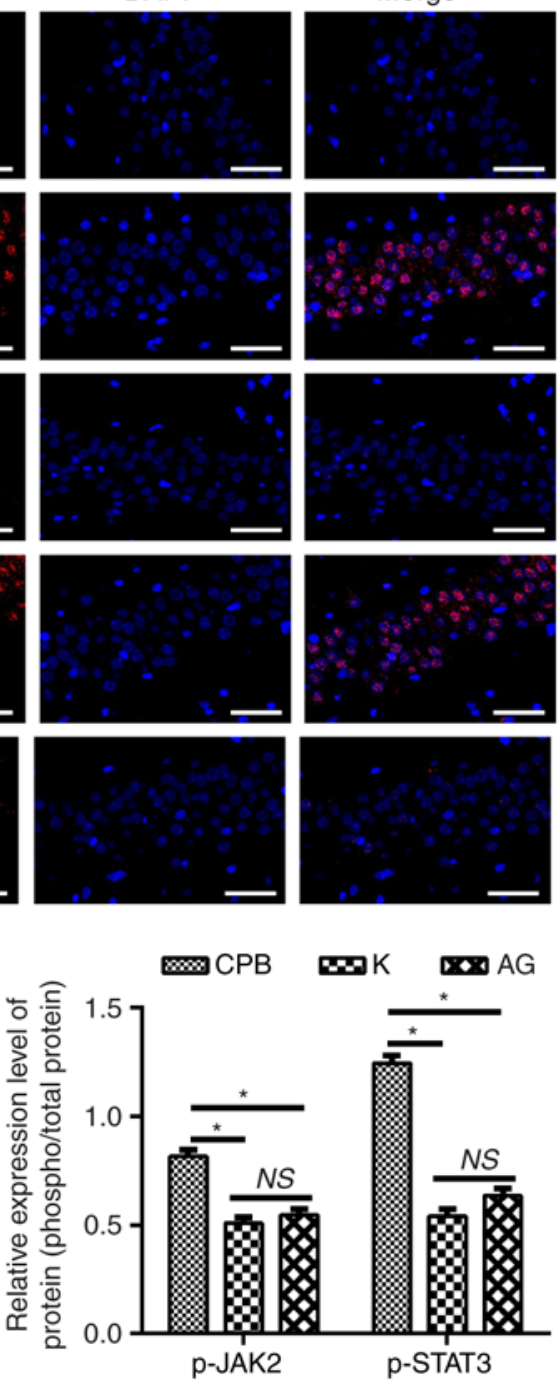
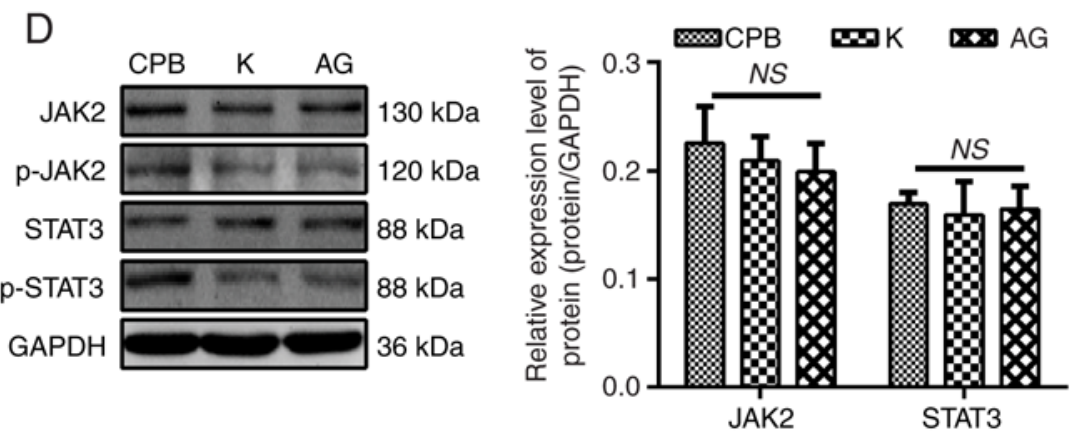

Figure 5. Continued. (C) JAK2, p-JAK2, STAT3 and p-STAT3 in the hippocampus were detected by immunofluorescence (scale bar=50 $\mu$ m). (D) Levels of JAK2, p-JAK2, STAT3 and p-STAT3 in the hippocampus were detected by western blotting. *P<0.05. CPB, cardiopulmonary bypass; KOR, kappa opioid receptor; NSE, neuron-specific enolase; K, KOR agonist + CPB; NK, KOR agonist + norbinaltorphimine + CPB; AG, KOR agonist + JAK2-STAT3 specific pathway inhibitor + CPB; AG, KOR agonist + JAK2-STAT3 specific pathway inhibitor + CPB; JAK2, Janus kinase 2; STAT3, signal transducer and activators of transcription 3; p-, phosphorylated; ns, not significant.

in the $\mathrm{K}$ group, the hippocampus of rats in the AG group was still damaged, with some disordered cells, which exhibited increasing astrocyte and vascular proliferation (Fig. 3A). The levels of p-JAK2 and p-STAT3 in the hippocampus of the AG group were significantly decreased compared with those in the $\mathrm{CPB}$ group $(\mathrm{P}<0.05)$. On comparing the $\mathrm{K}$ group and $\mathrm{AG}$ group, the difference was not statistically significant $(\mathrm{P}>0.05)$. There were no significant differences in the levels of JAK2 and STAT3 among the CPB, K and AG groups. Therefore, KOR agonists may improve POCD in CPB rats through the JAK2/STAT3 signaling pathway (Fig. 5C and D).

\section{Discussion}

In the present study, it was found that KOR agonists significantly improved cognitive dysfunction in CPB rats. S-100 $\beta$ and NSE detection showed that the KOR agonists alleviated brain damage in the CPB rats, and this result was reversed by KOR antagonists. KOR agonists significantly the reduced the inflammatory response and oxidative stress on ELISA detection. The KOR agonists were also detected to attenuate hippocampal neuronal apoptosis, as shown by TUNEL staining and western blotting, and downregulated the levels of p-JAK2, STAT3 and p-STAT3 compared with those in the CPB group. These results indicate that KOR agonists can improve cognitive dysfunction in POCD of CPB rats by inhibiting the JAK2/STAT3 signaling pathway. CPB technology provides an irreplaceable tool for doctors performing surgery (19). With the advancement of clinical medicine, CPB has been increasingly expanded into an important technology in clinical medicine $(20,21)$. However, with the increasing application of this technology, increasing complications are also being exposed (22). The perioperative period of CPB open-heart surgery, in-hospital mortality rates and postoperative complications have all decreased significantly. Only the incidence of neurological impairment has not declined, and neurological impairment can significantly increase in the perioperative period, with various complications and postoperative 
mortality, in addition to prolonged hospital admissions and an increase in the economic burden of patients. Permanent nerve injury not only reduces patient quality of life, but also requires re-admission to hospital and may even lead to death (23).

In the present study, the neurological function score and water maze test performance were assessed in in a POCD model in CPB rats. H\&E and TUNEL staining were used to observe the hippocampus. Oxidative stress factors, brain injury markers, inflammatory factors, apoptosis and JAK2/STAT3 signaling pathway-associated proteins were examined to investigate the role of KOR agonists in the development and progress of POCD in CPB rats. A novel treatment for POCD was provided and its mechanism was examined.

The results of the study showed that KOS may be a suitable drug target in POCD therapy. In addition to central nervous tissues, KOR is expressed in the hippocampal dentate gyrus, hypothalamus, certain thalamic nuclei, cerebral cortex, caudate nucleus, olfactory bulb, nucleus accumbens and spinal cords in rats (24-26). U50488H, a specific KOR agonist, blocks the transport of acetylcholine through the KOR-mediated opioid nervous system, which inhibits the reduction of acetylcholine release caused by mecamylamine (an $\mathrm{N}$-cholinoceptor-blocking drug), thus reversing the learning and memory damage caused by mecamylamine $(27,28)$. It has been shown that $\kappa$ agonists can improve memory damage caused by $\mu$ agonists; their effect is not only opposite to the effect of $\mu$ receptor agonists, but they also regulate components of the $\mu$ system, such as anti-nociceptive effects (29). Studies on KOR intervention (U50488H) in ischemia-induced hippocampal nerve injury have shown that the agonist can significantly reduce cognitive dysfunction (30). The present study confirmed that KOR agonists in CPB rats can inhibit the inflammatory response, reduce oxidative stress, inhibit neuronal apoptosis and improve brain damage, thus reducing the occurrence and development of POCD in CPB rats.

The JAK2/STAT3 signaling pathway is an important pathway for the cholinergic anti-inflammatory pathway (CAP) (31). With expanding research on CAP, its protection of the brain has attracted attention. Studies have shown that the anti-inflammatory effects of JAK2/STAT3 are activated when the core $\alpha 7$ nicotinic acetylcholine receptor $(\alpha 7 \mathrm{nAchR})$ of the CAP is activated $(32,33)$. In addition to its involvement in the inflammatory response, the JAK2/STAT3 signaling pathway is involved in the anti-inflammatory response following a7nAchR activation $(34,35)$. Studies have confirmed that the JAK2/STAT3 signaling pathway is involved in the anti-apoptotic process of cerebral ischemic neurons $(36,37)$.

In the present study, it was shown that KOR agonists can improve POCD of CPB rats via the phosphorylation JAK2 and STAT3, rather than affecting their expression. The phosphorylation or overexpression of JAK2 may be a mechanism of brain damage (16). Reducing JAK2/STAT3 phosphorylation can decrease neuronal death, narrow infarct size and prevent post-ischemic damage of nerve cells. Wang et al reported a significant neuroprotective effect by reducing the phosphorylation of STAT3 following cerebral ischemia through RNA interference (38). Others have found that electroacupuncture stimulation of focal cerebral ischemia at the Baihui acupoint and Dazhui acupoint in rats relieved nerve function deficit by reducing the expression of JAK2, preventing abnormal
JAK2 activation and downregulating the phosphorylation of STAT3 (37).

In conclusion, the findings of the present study suggest that KOR agonists provide neuroprotective effects against POCD brain damage in CPB rats, which is partially mediated by inhibition of the JAK2/STAT3 pathway. The findings regarding the KOR agonist-mediated molecular mechanisms and signaling pathways provide novel insight into, and a novel therapeutic target for, POCD brain damage. Studies in the future should focus on other possible relationships between JAK2/STAT3 and PI3K/AKT/mTOR in the action of KOR agonists in POCD brain damage.

\section{Acknowledgements}

Not applicable.

\section{Funding}

This study was supported by the Liaoning Natural Science Foundation (grant. no. 201602790) and the National Natural Science Foundation of China (grant. no. 81471121).

\section{Availability of data and materials}

The datasets used and/or analyzed during the present study are available from the corresponding author on reasonable request.

\section{Authors' contributions}

XL, YS and YD conceived and designed the study and drafted the manuscript. XL, YS, QJ and DS performed experiments and interpreted the results. QJ and DS analyzed the data. YS and YD contributed to acquisition of funding support. All authors read and approved the final manuscript.

\section{Ethics approval and consent to participate}

All animal protocols were approved by the Experimental Animal Ethics Committee of the General Hospital of Northern Theater Command (no. GHNTC2018018).

\section{Patient consent for publication}

Not applicable.

\section{Competing interests}

The authors declare that they have no competing interests.

\section{References}

1. Arrowsmith JE, Grocott HP, Reves JG and Newman MF: Central nervous system complications of cardiac surgery. $\mathrm{Br}$ J Anaesth 84: 378-393, 2000.

2. Evered L, Scott DA, Silbert B and Maruff P: Postoperative cognitive dysfunction is independent of type of surgery and anesthetic. Anesth Analg 112: 1179-1185, 2011.

3. Steinmetz J and Rasmussen LS: Peri-operative cognitive dysfunction and protection. Anaesthesia 71 (Suppl 1): S58-S63, 2016.

4. Noctor G, Lelarge-Trouverie C and Mhamdi A: The metabolomics of oxidative stress. Phytochemistry 112: 33-53, 2015. 
5. Zakkar M, Guida G, Suleiman MS and Angelini GD Cardiopulmonary bypass and oxidative stress. Oxid Med Cell Longev 2015: 189863, 2015.

6. Yang L, Shah K, Wang H, Karamyan VT and Abbruscato TJ: Characterization of neuroprotective effects of biphalin, an opioid receptor agonist, in a model of focal brain ischemia. J Pharmacol Exp Ther 339: 499-508, 2011

7. Shen J, Sun LN, Wu LP and Xia Q: Mito K(ATP) and kappa-opioid receptor mediate the neuroprotective effect of limb ischemic post-conditioning on rat brain ischemia/reperfusion injury. Zhongguo Ying Yong Sheng Li Xue Za Zhi 25: 368-372, 2009 (In Chinese).

8. Hiramatsu M, Murai M and Kameyama T: Different modulation of cholinergic neuronal systems by dynorphin A (1-13) in carbon monoxide-exposed mice. Biochem Pharmacol 57: 1321-1329, 1999

9. Olianas MC, Dedoni S, Ambu R and Onali P: Agonist activity of $\mathrm{N}$-desmethylclozapine at delta-opioid receptors of human frontal cortex. Eur J Pharmacol 607: 96-101, 2009.

10. Fei R, Zhang Y, Wang S, Xiang T and Chen W: $\alpha 7$ nicotinic acetylcholine receptor in tumor-associated macrophages inhibits colorectal cancer metastasis through the JAK2/STAT3 signaling pathway. Oncol Rep 38: 2619-2628, 2017.

11. Shi S, Liang D, Bao M, Xie Y, Xu W, Wang L, Wang Z and Qiao Z: Gx-50 inhibits neuroinflammation via $\alpha 7 \mathrm{nAChR}$ activation of the JAK2/STAT3 and PI3K/AKT pathways. J Alzheimers Dis 50: 859-871, 2016.

12. Hu GQ, Du X, Li YJ, Gao XQ, Chen BQ and Yu L: Inhibition of cerebral ischemia/reperfusion injury-induced apoptosis: Nicotiflorin and JAK2/STAT3 pathway. Neural Regen Res 12: 96-102, 2017.

13. Silverman J and Muir WW III: A review of laboratory animal anesthesia with chloral hydrate and chloralose. Lab Anim Sci 43 : 210-216, 1993.

14. Garcia JH, Wagner S, Liu KF and Hu XJ: Neurological deficit and extent of neuronal necrosis attributable to middle cerebral artery occlusion in rats. Statistical validation. Stroke 26: 627-634, discussion 635, 1995.

15. Bederson JB, Pitts LH, Tsuji M, Nishimura MC, Davis RL and Bartkowski H: Rat middle cerebral artery occlusion: Evaluation of the model and development of a neurologic examination. Stroke 17: 472-476, 1986.

16. Satriotomo I, Bowen KK and Vemuganti R: JAK2 and STAT3 activation contributes to neuronal damage following transient focal cerebral ischemia. J Neurochem 98: 1353-1368, 2006.

17. Lu Y,Zhou J, Xu C, Lin H, Xiao J, Wang Z and Yang B: JAK/STAT and PI3K/AKT pathways form a mutual transactivation loop and afford resistance to oxidative stress-induced apoptosis in cardiomyocytes. Cell Physiol Biochem 21: 305-314, 2008.

18. Xie HF, Xu RX, Wei JP, Jiang XD and Liu ZH: P-JAK2 and P-STAT3 protein expression and cell apoptosis following focal cerebral ischemia-reperfusion injury in rats. Nan Fang Yi Ke Da Xue Xue Bao 27: 208-211, 218, 2007 (In Chinese).

19. Katz MG, Fargnoli AS, Yarnall C, Perez A, Isidro A, Hajjar RJ and Bridges CR: Technique of complete heart isolation with continuous cardiac perfusion during cardiopulmonary bypass: New opportunities for gene therapy. J Extra Corpor Technol 50: 193-198, 2018.

20. Dimarakis I: Miniaturized cardiopulmonary bypass in adult cardiac surgery: A clinical update. Expert Rev Cardiovasc Ther 14: 1245-1250, 2016.

21. Melchior RW, Sutton SW, Harris W and Dalton HJ: Evolution of membrane oxygenator technology for utilization during pediatric cardiopulmonary bypass. Pediatric Health Med Ther 7: 45-56, 2016.

22. Sukumaran V, Tsuchimochi H, Fujii Y, Hosoda H, Kangawa K, Akiyama T, Shirai M, Tatsumi E and Pearson JT: Ghrelin Pre-treatment attenuates local oxidative stress and end organ damage during cardiopulmonary bypass in anesthetized rats. Front Physiol 9: 196, 2018.

23. Wimmer-Greinecker G, Matheis G, Brieden M, Dietrich M, Oremek G, Westphal K, Winkelmann BR and Moritz A: Neuropsychological changes after cardiopulmonary bypass for coronary artery bypass grafting. Thorac Cardiovasc Surg 46 207-212, 1998

24. Ardianto C, Yonemochi N, Yamamoto S, Yang L, Takenoya F, Shioda S, Nagase H, Ikeda H and Kamei J: Opioid systems in the lateral hypothalamus regulate feeding behavior through orexin and GABA neurons. Neuroscience 320: 183-193, 2016.
25. Minowa S, Ishihara S, Tsuchiya S, Horie S, Watanabe K and MurayamaT:Involvement of glutamateand gamma-amino-butyric acid receptor systems on gastric acid secretion induced by activation of kappa-opioid receptors in the central nervous system in rats. Br J Pharmacol 138: 1049-1058, 2003.

26. Terman GW, Drake CT, Simmons ML, Milner TA and Chavkin C: Opioid modulation of recurrent excitation in the hippocampal dentate gyrus. J Neurosci 20: 4379-4388, 2000.

27. Wang Q, Sun Y, Li J, Xing W, Zhang S, Gu X, Feng N, Zhao L, Fan R, Wang Y, et al: Quaternary ammonium salt of U50488H, a new K-opioid receptor agonist, protects rat heart against ischemia/reperfusion injury. Eur J Pharmacol 737: 177-184, 2014.

28. Tong G, Zhang B, Zhou X, Zhao J, Sun Z, Tao Y, Pei J and Zhang W: Kappa-opioid agonist U50,488H-mediated protection against heart failure following myocardial ischemia/reperfusion: Dual roles of heme oxygenase-1. Cell Physiol Biochem 39: 2158-2172, 2016.

29. Cheng MF, Ou LC, Chen SC, Chang WT, Law PY, Loh HH, Chao YS, Shih C, Yeh SH and Ueng SH: Discovery, structure-activity relationship studies, and anti-nociceptive effects of 1-phenyl-3,6,6-trimethyl-1,5,6,7-tetrahydro-4H-indazol-4-one as novel opioid receptor agonists. Bioorg Med Chem 22: 4694-4703, 2014.

30. Takahashi K, Nakagawasai O, Sugawara M, Sato A, Nemoto W, Tadano $\mathrm{T}$ and Tan-No K: Kappa opioid receptor agonist administration in olfactory bulbectomized mice restores cognitive impairment through cholinergic neuron activation. Biol Pharm Bull 41: 957-960, 2018.

31. Chatterjee PK, Al-Abed Y, Sherry B and Metz CN: Cholinergic agonists regulate JAK2/STAT3 signaling to suppress endothelial cell activation. Am J Physiol Cell Physiol 297: C1294-C1306, 2009.

32. Li T, Wu S, Zhang H, Wang Y, Luo H, Zuo X and Xiao X: Activation of nicotinic receptors inhibits TNF- $\alpha$-induced production of pro-inflammatory mediators through the JAK2/STAT3 signaling pathway in fibroblast-like synoviocytes. Inflammation 38: 1424-1433, 2015.

33. Yang YH, Li DL, Bi XY, Sun L, Yu XJ, Fang HL, Miao Y, Zhao M, He X, Liu JJ and Zang WJ: Acetylcholine inhibits LPS-induced MMP-9 production and cell migration via the $\alpha 7$ nAChR-JAK2/STAT3 pathway in RAW264.7 cells. Cell Physiol Biochem 36: 2025-2038, 2015.

34. Maldifassi MC, Atienza G, Arnalich F, López-Collazo E, Cedillo JL, Martín-Sánchez C, Bordas A, Renart J and Montiel C: A new IRAK-M-mediated mechanism implicated in the anti-inflammatory effect of nicotine via $\alpha 7$ nicotinic receptors in human macrophages. PLoS One 9: e108397, 2014.

35. Zhang W, Sun Q, Gao X, Jiang Y,Li R and Ye J: Anti-inflammation of spirocyclopiperazinium salt compound LXM-10 targeting $\alpha 7$ nAChR and M4 mAChR and inhibiting JAK2/STAT3 pathway in rats. PLoS One 8: e66895, 2013.

36. Chen B, Yang L, Chen J, Chen Y, Zhang L, Wang L, Li X, $\mathrm{Li} \mathrm{Y}$ and $\mathrm{Yu} \mathrm{H}$ : Inhibition of connexin43 hemichannels with Gap19 protects cerebral ischemia/reperfusion injury via the JAK2/STAT3 pathway in mice. Brain Res Bull 146: 124-135, 2019.

37. Xu H,Zhang YM, Sun H, Chen SH and Si YK: Electroacupuncture at GV20 and ST36 exerts neuroprotective effects via the EPO-mediated JAK2/STAT3 pathway in cerebral ischemic rats. Evid Based Complement Alternat Med 2017: 6027421, 2017.

38. Wang F, Li M, Li X, Kinden R, Zhou H, Guo F, Wang Q and Xiong L: 2-Arachidonylglycerol protects primary astrocytes exposed to oxygen-glucose deprivation through a blockade of NDRG2 signaling and STAT3 phosphorylation. Rejuvenation Res 19: 215-222, 2016.

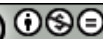

This work is licensed under a Creative Commons Attribution-NonCommercial-NoDerivatives 4.0 International (CC BY-NC-ND 4.0) License. 